Check for updates

Cite this: RSC Adv., 2019, 9, 35862

Received 20th April 2019

Accepted 20th October 2019

DOI: $10.1039 / c 9 r a 02958 c$

rsc.li/rsc-advances

\section{DFT calculation and analysis of the gas sensing mechanism of methoxy propanol on Ag decorated $\mathrm{SnO}_{2}$ (110) surface}

\author{
Meihua Li, (D) *ac Huichao Zhu, (iD b Guangfen Wei, ${ }^{\text {ac }}$ Aixiang $\mathrm{He}^{\text {ac }}$ and Yanli Liu ${ }^{\text {ac }}$
}

Methoxy propanol has been widely used in modern industry and consumer products. Inhalation or skin exposure to methoxy propanol for a long period would bring about safety challenges on human habitat and health. Ag decorated $\mathrm{SnO}_{2}$ mesoporous material has been synthesized and shown to exhibit high sensitivity and good selectivity to methoxy propanol among other interferential VOC gases. Density Functional Theory study were conducted to yield insight into the surface-adsorbate interactions and therefore the gas sensing improvement mechanism by presenting accurate energetic and electronic properties for the $\mathrm{Ag} / \mathrm{SnO}_{2}$ system. Firstly, an electron transfer model on $\mathrm{Ag}$ and $\mathrm{SnO}_{2}$ grain interface was put forward to illustrate the methoxy propanol gas sensing mechanism. Then, a three-layer adsorption model (TLAM) was proposed to investigate methoxy propanol gas sensing properties on a $\mathrm{SnO}_{2}$ (110) surface. In the TLAM method, taking $\mathrm{SnO}_{2}$ (110) surface for the basis, layer 1 illustrates the decoration of metal $\mathrm{Ag}$ on $\mathrm{SnO}_{2}$ (110) surface. Layer 2 represents the adsorption of molecular oxygen on metal $\mathrm{Ag}$ decorated $\mathrm{SnO}_{2}$ (110) surface. Layer 3 indicates the adsorption of methoxy propanol, and for comparison, three other VOC gases (namely, ethanol, isopropanol and p-xylene) on Ag decorated $\mathrm{SnO}_{2}$ (110) surface with oxygen species pre-adsorbed consecutively. All the adsorption processes were calculated by means of Density Functional Theory method; the adsorption energy, net charge transfer, DOS, PDOS and also experimental data were utilized to investigate the methoxy propanol gas sensing mechanism on Ag decorated $\mathrm{SnO}_{2}$ (110) surface with oxygen species pre-adsorbed.

\section{Introduction}

With the extensive industrial applications and natural/ anthropogenic emissions of chemical species, volatile organic compounds (VOCs) have become ubiquitous in our environment. Several kinds of VOCs emitted from human life commodities have put forward safety challenges on human habitats and have been reported to have slow yet long term pathogenic effects on human health. ${ }^{1}$ Propylene glycol ethers, among which methoxy propanol, have been widely used in modern industry and consumer products. It is reported that the content of methoxy propanol is mostly above $50 \%$ in paints, inks, solvents, varnishes, hardeners, auxiliary materials and so on. ${ }^{2}$ Long-term inhalation or skin exposure to methoxy propanol at considerably high concentrations could cause inflammatory changes in healthy subjects' nasal epithelium or impair the mucociliary transport system. ${ }^{3}$ The MAK value for methoxy

${ }^{a}$ School of Information and Electronic Engineering, Shandong Technology and Business University, Yantai 264005, China.E-mail: limeihua@sdtbu.edu.cn

${ }^{b}$ School of Biomedical Engineering, Dalian University of Technology, Dalian 116024, China

${ }^{c}$ Key Laboratory of Sensing Technology and Control in Universities of Shandong, Shandong Technology and Business University, Yantai 264005, China propanol has been set as $100 \mathrm{ppm}$ to protect human bodies from its irritating effects; ${ }^{4}$ in other words, once the concentration of methoxy propanol exceeds 100 ppm, systemic toxicity effects would come into sight. ${ }^{5}$ Thus, a highly sensitive and selective detection of methoxy propanol becomes very essential to human health and safety, especially for the workers who are exposed at workplaces where this chemical compound is largely used or produced.

As a metal oxide semiconductor material with a broad bandgap $\left(E_{\mathrm{g}}=3.6 \mathrm{eV}\right),{ }^{6,7} \mathrm{SnO}_{2}$ has always attracted wide interest in the field of gas sensors due to its good conductivity, thermal stability and surface reactivity. On the one hand, the interaction between a target gas and $\mathrm{SnO}_{2}$ has been widely investigated experimentally: ${ }^{8-12}$ it has been found that the gas sensing properties of $\mathrm{SnO}_{2}$ mainly depend on its surficial chemical transduction, which involves several stages including the adsorption of the target species and charge transfer between the adsorbate and the sensing material. On the other hand, doping with noble metals is an efficient way to improve the gas sensing properties of pure $\mathrm{SnO}_{2}$, for which $\mathrm{Ag}$ is an excellent candidate. ${ }^{13-16}$ Although it is well known that Ag can improve the gas sensing performance of $\mathrm{SnO}_{2}$, the enhancement mechanism remains unclear. It has been generally ascribed to a catalytic effect; however, the atomic configuration and chemical 
environment at the material surface, which might have a strong effect on the sensing properties, are hard to characterize, hence posing an essential challenge to the understanding of the gas sensing mechanism of the $\mathrm{Ag} / \mathrm{SnO}_{2}$ system.

Density Functional Theory (DFT) studies yield insight into atomic geometries and the nature of chemical bonding, and therefore can provide a valuable tool for understanding surfaceadsorbate interactions by presenting their accurate energetic and electronic properties. ${ }^{\mathbf{1 7 - 3 0}}$ However, to the best of our knowledge, DFT calculations and analysis of the methoxy propanol gas sensing performance of a $\mathrm{Ag}$ decorated $\mathrm{SnO}_{2}$ (110) surface have rarely been conducted. In one of our previous research, ${ }^{31}$ a $\mathrm{Ag}$ decorated $\mathrm{SnO}_{2}$ mesoporous material has been synthesized through a two-step synthetic route, and subsequently tested for the detection of VOC gases. This Ag-decorated $\mathrm{SnO}_{2}$ mesoporous material showed high sensitivity and good selectivity towards methoxy propanol among other interferential VOC gases.

In this work, an electron transfer model of a $\mathrm{Ag}$ and $\mathrm{SnO}_{2}$ grain interface is presented to illustrate the catalytic effect of Agdoped $\mathrm{SnO}_{2}$ for methoxy propanol gas sensing. Then, a threelayer adsorption model (TLAM) was proposed to investigate the mechanism of the VOC gas sensing performance of the $\mathrm{SnO}_{2}$ (110) surface. In our TLAM method, the decoration of $\mathrm{Ag}$ metal on the $\mathrm{SnO}_{2}$ (110) surface was first modelled and optimized in structure. Secondly, molecular oxygen adsorption configurations were proposed and the corresponding DFT calculations on the $\mathrm{Ag}$ decorated $\mathrm{SnO}_{2}$ (110) surface were conducted. Thirdly, the adsorption of methoxy propanol, and for comparison, three other VOC gases (namely, ethanol, isopropanol and $p$-xylene) on the Ag decorated $\mathrm{SnO}_{2}$ (110) surface with pre-adsorbed oxygen species were modelled and calculated consecutively. At last, according to the results from the calculations, the adsorption energy values, net charge transfer, DOS and PDOS were utilized to investigate the methoxy propanol gas sensing properties of the Ag-decorated $\mathrm{SnO}_{2}$ material.

\section{Methoxy propanol gas sensing properties and mechanism on the surface of a Ag decorated $\mathrm{SnO}_{2}$ material}

A two-step synthesis route was presented to prepare the $\mathrm{Ag}$ decorated $\mathrm{SnO}_{2}$ mesoporous material. Firstly, carbon nanospheres were obtained by hydrothermal synthesis, and then immersed in an absolute ethyl alcohol solution of $\mathrm{SnCl}_{4} \cdot 5 \mathrm{H}_{2} \mathrm{O}$. The mixture was centrifuged, washed and calcined to get a pure $\mathrm{SnO}_{2}$ material. Secondly, $\mathrm{AgNO}_{3}$ and ascorbic acid were introduced to implement the decoration of $\mathrm{Ag}$ nanoparticles on the porous $\mathrm{SnO}_{2}$ material through a redox reaction. A SEM image of the as-prepared material is shown in Fig. 1(a), where the mesoporous microstructure with a wall thickness of $70 \mathrm{~nm}$, and the decorating Ag nanoparticles with diameters of no more than $100 \mathrm{~nm}$ can be easily observed. Fig. 1(b) shows the elemental composition of the prepared material; a weight ratio of $1.62 \%$ for the Ag element further confirms the decoration of $\mathrm{Ag}$ onto the $\mathrm{SnO}_{2}$ material. Fig. 2 shows the cross response of our $\mathrm{Ag}$ decorated $\mathrm{SnO}_{2}$ material towards four typical VOC gases, namely, methoxy propanol, ethanol, isopropanol and $p$-xylene, with the response being defined as $R_{\mathrm{a}} / R_{\mathrm{g}}\left(R_{\mathrm{a}}\right.$ represents the sensor resistance in air, $R_{\mathrm{g}}$ represents the sensor resistance in the presence of the target gas). A relatively high response within a wide gas concentration range from $50 \mathrm{ppm}$ to $300 \mathrm{ppm}$ proves a higher detection selectivity for methoxy propanol compared to the other three gases. It can be seen that the response of the Ag/ $\mathrm{SnO}_{2}$ material to $100 \mathrm{ppm}$ methoxy propanol is $\sim 130$, and when the concentration increases to $150 \mathrm{ppm}, 200 \mathrm{ppm}$ and $250 \mathrm{ppm}$, the response accordingly increases to $\sim 580, \sim 920$ and $\sim 1000$, respectively. These data fully prove the high sensitivity and good selectivity of the $\mathrm{Ag} / \mathrm{SnO}_{2}$ material to methoxy propanol, and its potential in the detection of methoxy propanol. More detailed experimental description can be found in the literature. ${ }^{31}$
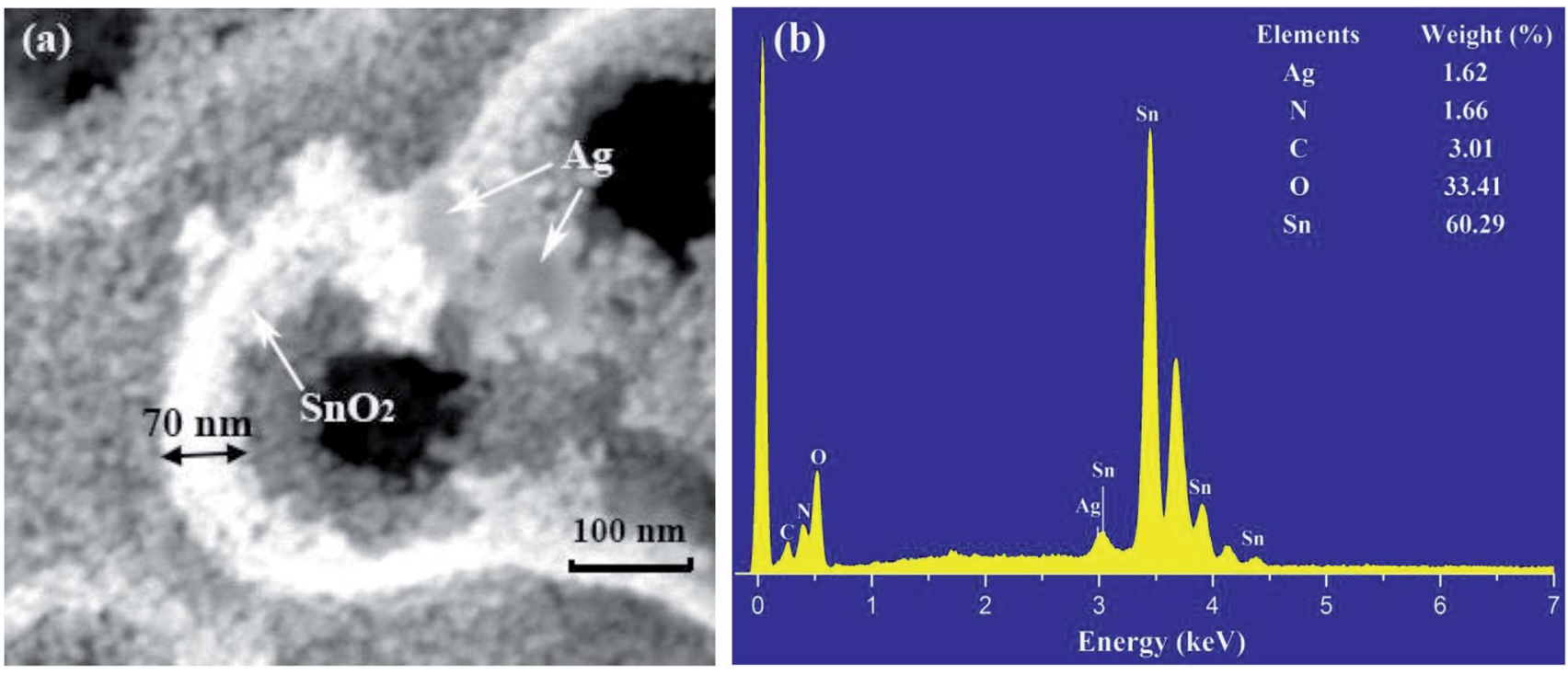

Fig. 1 (a) SEM image, (b) EDS spectrum of our Ag decorated $\mathrm{SnO}_{2}$ material. 


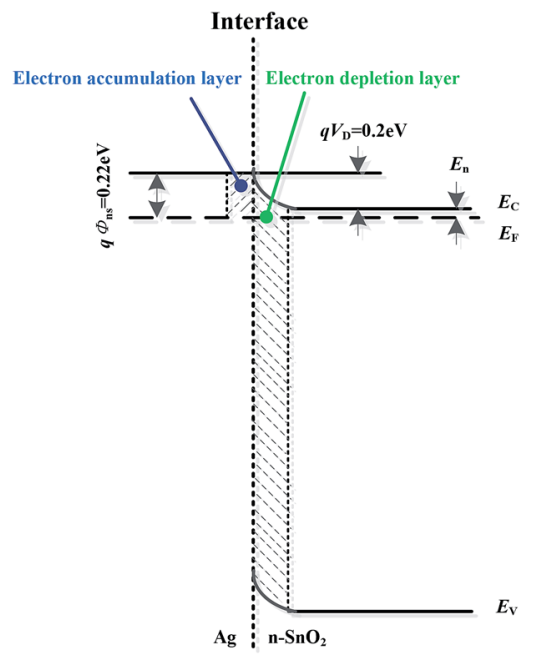

Fig. 2 Selectivity of the $\mathrm{Ag}$ decorated $\mathrm{SnO}_{2}$ material for $\mathrm{VOC}$ gases.

It is well known that the gas sensing mechanism of $\mathrm{SnO}_{2}$ is explained by the Surface Resistance Controlling Model. In general, when $\mathrm{SnO}_{2}$ sensors are exposed to air, atmospheric oxygen molecules adsorbed on the material's surface can grab electrons from the conduction band of $\mathrm{SnO}_{2}$ and turn into oxygen species such as $\mathrm{O}_{2}{ }^{-}, \mathrm{O}^{-}$or $\mathrm{O}^{2-} ;^{32-34}$ therefore, the material's surficial carrier concentration and electron mobility decrease, which results in a relevant increase in its sensor resistance. When reducing gases (methoxy propanol, ethanol, etc.) introduced, they reacted with the ionized oxygen species and released trapped electrons back onto the surface of $\mathrm{SnO}_{2}$. Consequently, the carrier concentration and electron mobility increased again, while the sensor resistance was correspondingly reduced. The larger the difference in the sensor resistance between the two cases, the higher the sensitivity of the gas sensor to a target gas.

As to $\mathrm{Ag}$ decorated $\mathrm{SnO}_{2}$ materials, the spill-over effect of the $\mathrm{Ag}$ metal might play a catalytic role to the contribution of electron quantity according to the well-established "chemical sensitization" mechanism. ${ }^{35-38}$ On the interface of $\mathrm{Ag}$ and $\mathrm{SnO}_{2}$

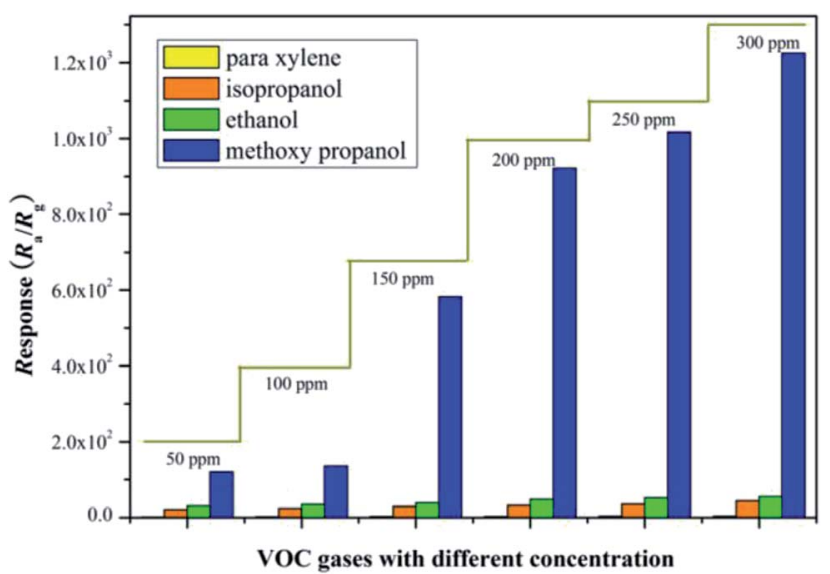

Fig. 3 Energy band on $\mathrm{Ag}$ and $\mathrm{SnO}_{2}$ grain boundary.
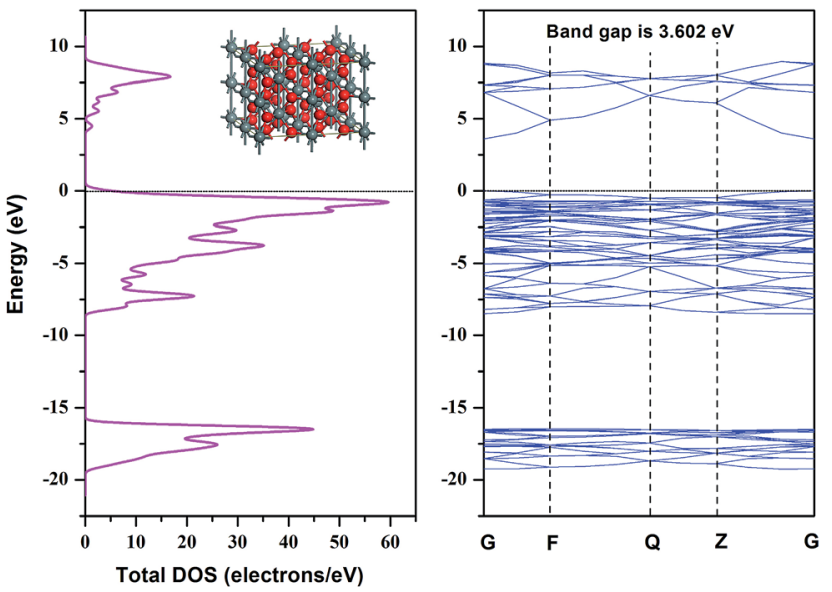

Fig. 4 DOS spectrogram and Band Structure of $\mathrm{SnO}_{2}$ super cell with 48 atoms.

grains, electrons transfer from $\mathrm{SnO}_{2}$ to $\mathrm{Ag}$ because the Fermi level of the former is higher than that of the latter. Once this transfer process reaches a kinematic equilibrium state, an electron accumulation layer and an electron depletion layer is generated on each side of the grain boundary, as shown in Fig. 3. The barrier height on the side of $\mathrm{SnO}_{2}$ is

$$
q V_{\mathrm{D}}=W_{1}-W_{2}=0.2 \mathrm{eV}
$$

and the barrier height on the side of $\mathrm{Ag}$ is

$$
q \Phi_{\mathrm{ns}}=q V_{\mathrm{D}}+E_{n}=W_{1}-W_{2}+E_{n}=W_{1-\chi}=0.22 \mathrm{eV}
$$

where $W_{1}$ is the work function of $\mathrm{Ag}$ with a value of $4.72 \mathrm{eV}, W_{2}$ is the work function of $\mathrm{SnO}_{2}$ with a value of $4.52 \mathrm{eV}$, and $\chi$ is the electronic affinity of $\mathrm{SnO}_{2}$ with a value of $4.5 \mathrm{eV}$.

This catalytic effect of $\mathrm{Ag}$ might further spill electrons on the surface of these materials and promote the ionization of molecular oxygen; so the electrons withdrawn from the $\mathrm{Ag} / \mathrm{SnO}_{2}$ composite materials become amplified and much faster than
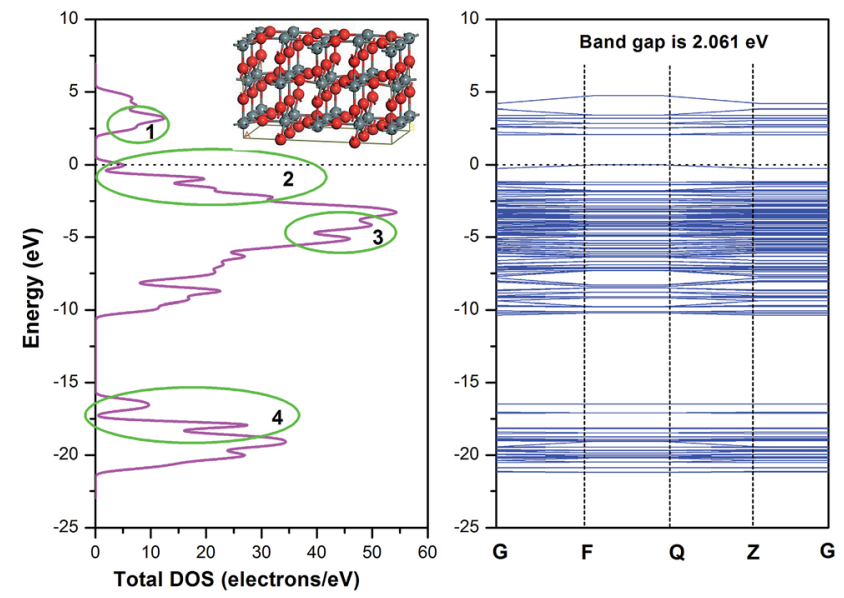

Fig. 5 DOS spectrogram and Band Structure of $\mathrm{SnO}_{2}$ (110) surface. 
(a)

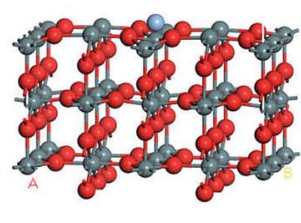

(b)

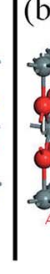

$\mathrm{Sn}$

O

$\mathrm{Ag}$

Fig. 6 Decoration of $\mathrm{Ag}$ on $\mathrm{SnO}_{2}$ (110) surface, (a) $\mathrm{Ag}$ is above $\mathrm{Sn}$ atom, (b) $\mathrm{Ag}$ is above $\mathrm{O}$ atom.

those withdrawn from pure $\mathrm{SnO}_{2}$. Once the material was exposed to methoxy propanol, reactions between methoxy propanol and oxygen species were activated. As the quantity of released electrons after these chemical reactions increased, the sensitivity of $\mathrm{Ag} / \mathrm{SnO}_{2}$ composite materials also increased. The typical reactions of methoxy propanol (chemical formula: $\mathrm{CH}_{3} \mathrm{CHOHCH}_{2} \mathrm{OCH}_{3}$ ) and ethanol (chemical formula: $\mathrm{C}_{2} \mathrm{H}_{5} \mathrm{OH}$ ) with ionized oxygen species are as follows:

$$
\begin{aligned}
\mathrm{CH}_{3} \mathrm{CHOHCH}_{2} \mathrm{OCH}_{3}+(13 / 2) \mathrm{O}_{2}{ }^{-} \rightarrow 4 \mathrm{CO}_{2} \uparrow & +5 \mathrm{H}_{2} \mathrm{O} \\
& +13 \mathrm{e}^{-} \\
\mathrm{C}_{2} \mathrm{H}_{5} \mathrm{OH}+3 \mathrm{O}_{2}{ }^{-} \rightarrow 2 \mathrm{CO}_{2} \uparrow & +3 \mathrm{H}_{2} \mathrm{O}+6 \mathrm{e}^{-}
\end{aligned}
$$

It is obvious that as long as there are sufficient adsorbed oxygen species, methoxy propanol will react with them and release more electrons back to the material surface than in the case of ethanol. This might be the main aspect of the superior sensing ability of methoxy propanol.

\section{Theoretical methods and computing platform}

Based on the above-mentioned Surface Resistance Controlling Theory applied to our $\mathrm{SnO}_{2}$ gas sensing material, a three-layer adsorption model (TLAM) was proposed to investigate the methoxy propanol gas sensing performance of the $\mathrm{SnO}_{2}$ (110) surface. In our TLAM method, the $\mathrm{SnO}_{2}$ (110) surface is considered the base of the composite; layer 1 refers to the decoration of Ag metal on $\mathrm{SnO}_{2}$, and layer 2 represents the adsorption of molecular oxygen on the $\mathrm{Ag}$ metal decorated $\mathrm{SnO}_{2}$
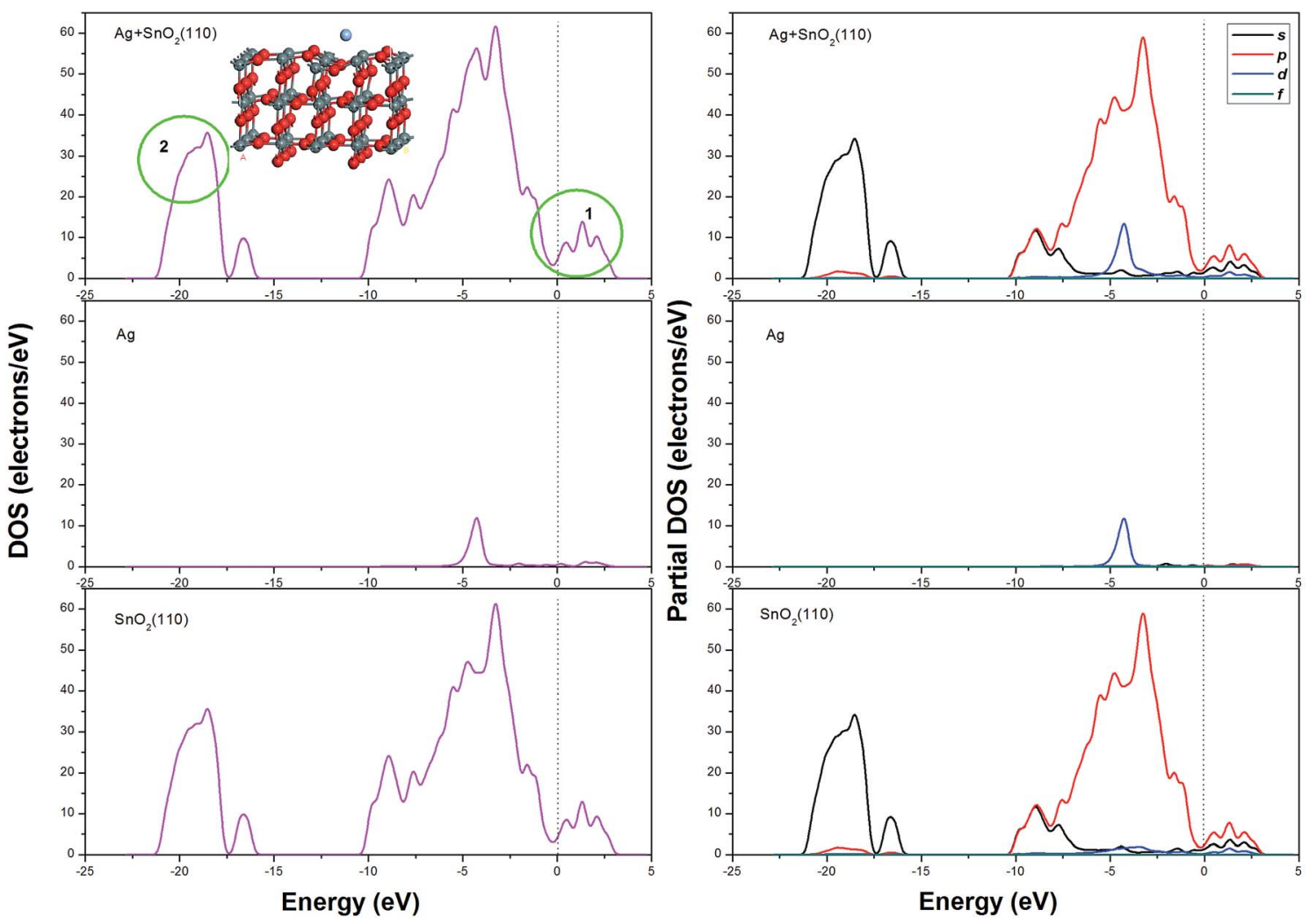

Fig. 7 DOS and PDOS spectrogram of Ag decorated $\mathrm{SnO}_{2}$ (110) surface. 
(110) surface. Layer 3 indicates the adsorbed methoxy propanol, and for comparison, three other VOC gases (namely, ethanol, isopropanol and $p$-xylene) on the $\mathrm{Ag}$ decorated $\mathrm{SnO}_{2}$ (110) surface where the oxygen species were pre-adsorbed.

In this paper, the DFT method was employed to carry out the calculations. All DFT calculations were implemented through the $\mathrm{DMol}^{3}$ package in the Materials Studio platform. ${ }^{39,40}$ The exchange and correlation energy calculations were performed by applying the Generalized Gradient Approximation through the Perdew-Burke-Ernzerhof method (GGA-PBE), ${ }^{41}$ which adopts double numerical basis sets polarization functions (DNP). The applied convergence criteria of optimal geometry were as follows: $1 \times 10^{-5} \mathrm{Ha}$ (energy), $0.002 \mathrm{Ha} \cdot \AA^{-1}$ (force) and $0.005 \AA$ (displacement). A $3 \times 1 \times 1$ Monkhorst-Pack $k$-point mesh was utilized for the Brillouin zone sampling and a $3 \times 1$ $\times 1$ Monkhorst-Pack grid was used to calculate the total energy and density of states (DOS). The Milliken population analysis $(\mathrm{MPA})^{42}$ method was used to calculate the charge transfer during the gas adsorption process.

\section{Results and discussion}

Layer 1 in TLAM method: modelling and optimization of $\mathrm{SnO}_{2}$ (110) surface with Ag decoration

Due to the minimum surface energy, $\mathrm{SnO}_{2}(110)$ is the most thermodynamically stable one among all crystal surfaces of

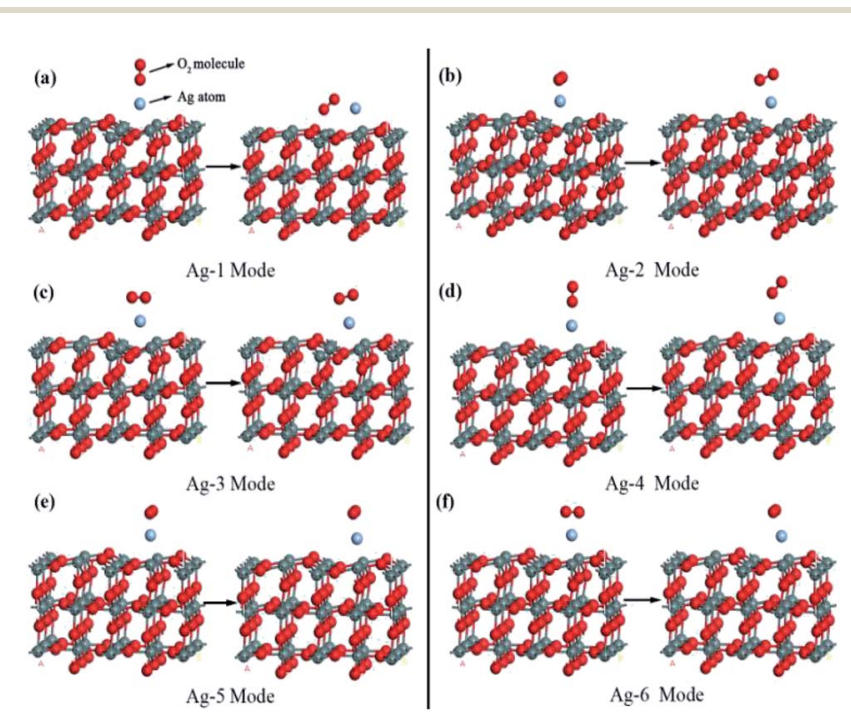

Fig. 8 Adsorption of $\mathrm{O}_{2}$ molecule on $\mathrm{Ag}$ decorated $\mathrm{SnO}_{2}(110)$ surface.
$\mathrm{SnO}_{2}$. In this section, the $\mathrm{SnO}_{2}$ atomic model with tetragonal structure (Pmnm(42)) was firstly built, and then a crystal model with 48 atoms in the super cell was established with lattice constants $a=b=4.73727 \AA, c=3.18638 \AA$. The DOS (Density of States) spectrogram and band structure of $\mathrm{SnO}_{2}$ super cell are shown in Fig. 4. It could be seen that the bandgap of the $\mathrm{SnO}_{2}$ material is $3.602 \mathrm{eV}$, which is in line with the experimental data, ${ }^{\mathbf{4 3 , 4 4}}$ and the valence bandwidth of $\mathrm{SnO}_{2}$ is $8.8 \mathrm{eV}$, coinciding with the experimental evidence. ${ }^{45}$ As the conduction band bottom and the valence band top are both at the zero point of the Brillouin zone ( $\mathrm{G}$ point), it could be inferred that the as-built $\mathrm{SnO}_{2}$ model belongs to a direct transition semiconductor.

The $\mathrm{SnO}_{2}$ (110) surface was cleaved from the as-optimized $\mathrm{SnO}_{2}$ bulk and a vacuum of $12 \AA$ was added to simulate the periodic boundary conditions. The calculated DOS spectrum and band structure of $\mathrm{SnO}_{2}$ (110) surface are shown in Fig. 5 . Compared with the bulk material, the band gap of $\mathrm{SnO}_{2}(110)$ was reduced to $2.061 \mathrm{eV}$, while the valence bandwidth was increased to $10.5 \mathrm{eV}$. Furthermore, there are four places (labelled as 1-4) where obvious changes could be observed: in place 1 , the peak shifted from $7.5 \mathrm{eV}$ to $3 \mathrm{eV}$; in place $2-4$, the DOS curve changed sharply and differentiated into several small peaks.

For the decoration of $\mathrm{Ag}$ on the $\mathrm{SnO}_{2}$ (110) surface, two kinds of configurations were considered; in one case, $\mathrm{Ag}$ atom was above $\mathrm{Sn}$ atom and in the other case $\mathrm{Ag}$ was above $\mathrm{O}$ atom, as shown in Fig. 6(a) and (b), respectively. In each configuration, the structure on the left of the arrow is the case before decoration and the right one is the case after decoration. The total energy of each optimized configurations has the same value of $-3538.727 \mathrm{Ha}$, and the net transfer charge of $\mathrm{Ag}$ atom is $-0.136 e$ and $-0.085 e$, respectively. The DOS and PDOS spectrum of Ag decorated $\mathrm{SnO}_{2}$ (110) surface are shown in Fig. 7; it is very interesting that the band gap has disappeared and the DOS curve in the conduction has differentiated into three small peaks (labelled as 1), which might imply an enhanced electric conductivity for $\mathrm{SnO}_{2}$ (110) surface to some extent. In addition, in the DOS fragment labelled as 2, the curve became smooth, which might imply that the energy distribution of electrons became gradual. A more detailed PDOS spectrum could be seen showing that this phenomenon was mainly attributed by $\mathrm{s}$ and $\mathrm{p}$ electrons of $\mathrm{SnO}_{2}$ (110). Comparing the DOS spectrum before and after Ag decoration, it is reasonable to deduce that the catalysis of $\mathrm{Ag}$ atom, especially the d electrons in $\mathrm{Ag}$, plays an

Table 1 Adsorption energy and transfer charge of oxygen species on metal Ag decorated $\mathrm{SnO}_{2}$ (110) surface

\begin{tabular}{llllr}
\hline Adsorption configuration & $\begin{array}{l}\text { Adsorption } \\
\text { energy }(\mathrm{eV})\end{array}$ & $\begin{array}{l}\mathrm{O}-\mathrm{O} \\
\text { bond length }(\AA)\end{array}$ & \multicolumn{2}{l}{$\begin{array}{l}\text { Transfer charge } \\
\text { of } \mathrm{O}_{2}(\mathrm{e})\end{array}$} \\
\hline $\mathrm{Ag}-1$ & -1.63 & 1.345 & -0.468 & 0.032 \\
$\mathrm{Ag}-2$ & -0.98 & 1.309 & -0.304 & -0.043 \\
$\mathrm{Ag}-3$ & -0.98 & 1.309 & -0.304 & -0.047 \\
Ag-4 & -0.98 & 1.279 & -0.253 & 0.016 \\
Ag-5 & -0.98 & 1.319 & -0.332 & 0.060 \\
Ag-6 & -0.98 & 1.320 & -0.336 & 0.058
\end{tabular}




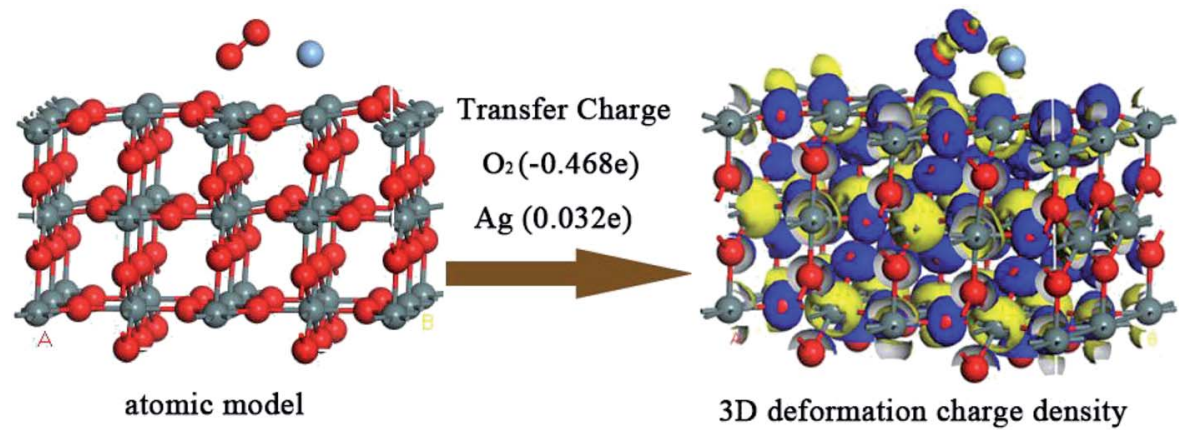

Fig. $93 \mathrm{D}$ deformation charge density of oxygen species pre-adsorbed and Ag decorated $\mathrm{SnO}_{2}$ (110) surface in Ag-1 configuration.

important role in the electrical conductivity changes of $\mathrm{SnO}_{2}$ (110) surface.

\section{Layer 2 in TLAM method: pre-adsorption of molecular oxygen on $\mathrm{Ag}$ decorated $\mathrm{SnO}_{2}(110)$ surface}

In order to understand the adsorption property of molecular oxygen on $\mathrm{Ag}$ decorated $\mathrm{SnO}_{2}$ (110) surface, six kinds of possible adsorption configurations (labelled as Ag-1 to Ag-6 in sequence) was built as shown as Fig. 8. In Ag-1, Ag-2 and Ag-3 configurations, molecular oxygen $\left(\mathrm{O}_{2}\right)$ was placed respectively perpendicularly to the $\mathrm{OA}$ axis, parallelly to the $\mathrm{OA}$ axis and parallelly to the $\mathrm{OB}$ axis, and all the three configurations take the optimized $\mathrm{Ag}$ decorated $\mathrm{SnO}_{2}$ (110) surface as shown in Fig. 6(a) as the adsorptive substrate. In Ag-4, Ag-5 and Ag-6 configurations, molecular $\mathrm{O}_{2}$ was placed respectively perpendicularly to the $\mathrm{OA}$ axis, parallelly to the $\mathrm{OA}$ axis and parallelly to the $\mathrm{OB}$ axis, and all the three configurations take the optimized Ag decorated $\mathrm{SnO}_{2}$ (110) surface as shown in Fig. 6(b) as the adsorptive substrate.

All calculations of six adsorption configurations have been carried out successively and the results are summarized in Table 1. There into, adsorption energy was adopted to judge the adsorption strength of molecular oxygen, which is defined as follows: ${ }^{46}$

$$
E_{\text {ads }}=E_{\text {adsorbate+surface }}-E_{\text {surface }}-E_{\text {adsorbate }}
$$

$E_{\text {ads }}$ is the adsorption energy, $E_{\text {adsorbate+surface }}$ represents the total energy of the whole adsorption system, $E_{\text {surface }}$ represents the initial energy of the adsorption surface and $E_{\text {adsorbate }}$
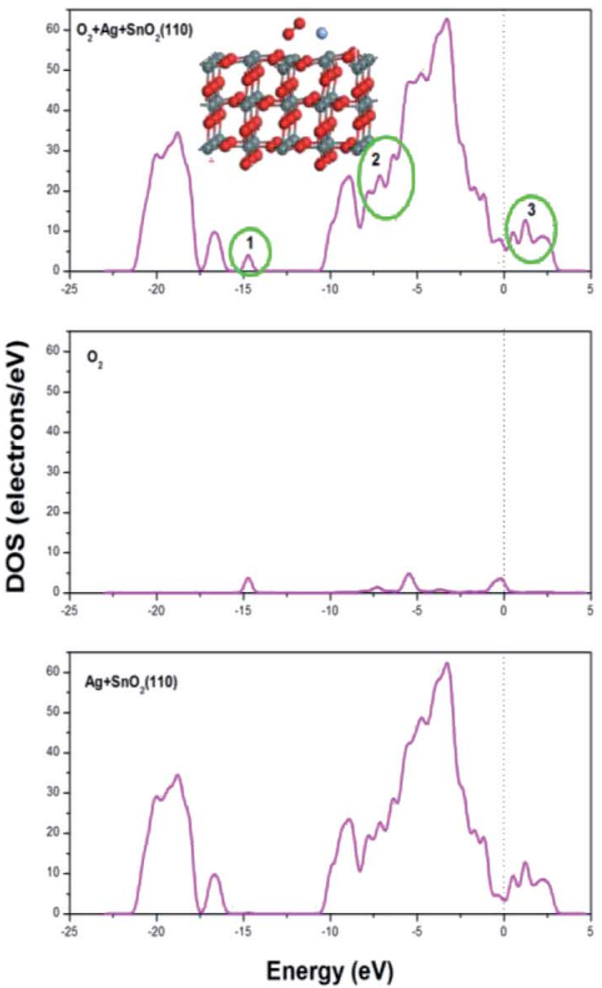
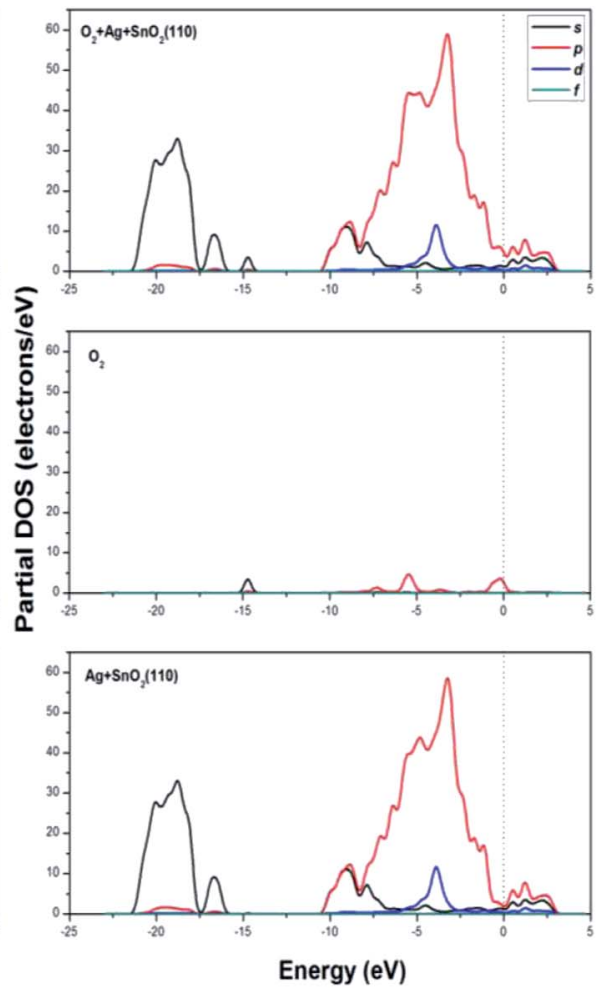

Fig. 10 DOS and PDOS spectrogram after $\mathrm{O}_{2}$ adsorption on $\mathrm{Ag}$ decorated $\mathrm{SnO}_{2}$ (110) surface. 
(a) (b)

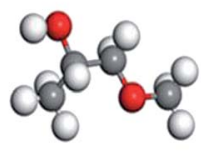

(c)

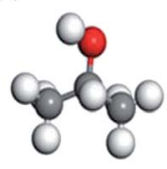

$\mathrm{H}$ atom (d)

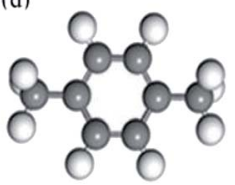

$\mathrm{O}$ atom
Fig. 11 Configurated molecular structure of methoxy propanol, ethanol, isopropanol and $p$-xylene.

represents the energy of the adsorbate. In general, $E_{\text {ads }}$ with a negative value indicates that the adsorption is exothermic and therefore the system after adsorption is energetically stable. ${ }^{47,48}$

It could be seen from Table 1 that, firstly, the adsorption energies of six configurations are all negative, which means that molecular oxygen could be adsorbed spontaneously on the $\mathrm{SnO}_{2}$ (110) surface. The absolute value of adsorption energy in Ag-1 configuration is the greatest, which means the strongest adsorption strength, and in other words, this kind of adsorption was most likely to take place. Secondly, after adsorption, the $\mathrm{O}-\mathrm{O}$ band length in oxygen species increased than the original $\mathrm{O}-\mathrm{O}$ band length $(1.226 \AA)$ before adsorption, and among them, the increment of $\mathrm{O}-\mathrm{O}$ band length in $\mathrm{Ag}-1$ configuration is the greatest. This case proves that the adsorption process would lead to a dissociation tendency of the two $\mathrm{O}$ atoms in oxygen species. Thirdly, oxygen species had negative net transfer charges in all adsorption configurations; Ag had negative charge in Ag-2 and $\mathrm{Ag}-3$ configurations, while the polarity of
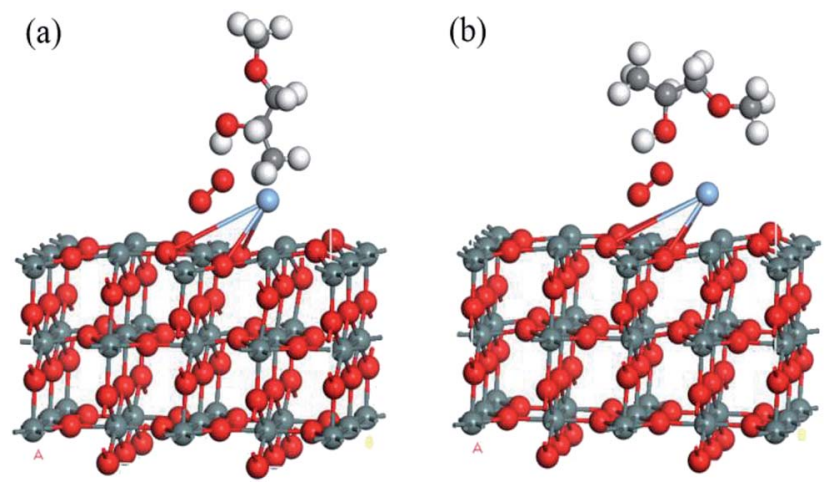

Fig. 12 Vertical and parallel adsorption configurations on oxygen species pre-adsorbed and $\mathrm{Ag}$ decorated $\mathrm{SnO}_{2}$ (110) surface of methoxy propanol. transfer charge in Ag-1, Ag-4, Ag-5 and Ag-6 configurations were positive.

Taking Ag-1 configuration for example, a three-dimensional (3D) plot of deformation charge density is shown in Fig. 9, where the blue regions represent electron trapping and yellow regions correspond to electron releasing; specifically, the transferred charge of $\mathrm{O}_{2}$ molecule is $-0.468 \mathrm{e}$, while that of $\mathrm{Ag}$ atom is $0.032 \mathrm{e}$. The DOS and PDOS spectra after $\mathrm{O}_{2}$ adsorption for Ag-1 configuration are shown in Fig. 10; it is obvious that a small DOS peak in the range of -15 to $-13 \mathrm{eV}$ (as labelled 1) attributed by $\mathrm{O}_{2}$ appeared, in places labelled as 2 and 3; the DOS value both increased, which implied the increase of electron energy and chemical activity in turn.

In short, in Ag-1 configuration, the adsorption of molecular oxygen on the $\mathrm{SnO}_{2}$ (110) surface would be the strongest and molecular oxygen would capture the maximum electrons from the material surface. Additionally, it is obvious that oxygen species moved closer to the $\mathrm{SnO}_{2}$ (110) surface in Ag-1 configuration, while in the other five configurations, oxygen species moved farther from the surface or showed almost no change, which implies more adsorption easiness in Ag-1 configuration. So, in the subsequent research, the oxygen species adsorbed surface in Ag-1 configuration would be taken as the adsorption substrate for the upcoming target gases.

Layer 3 in TLAM method: adsorption of methoxy propanol on Ag metal decorated $\mathrm{SnO}_{2}$ (110) surface with oxygen species pre-adsorbed

According to the traditional sensing theory, the chemical reaction between the target gas and pre-adsorbed oxygen species would cause the conductivity change through an electron exchange mechanism. In order to study the high sensitivity and good selectivity of the $\mathrm{Ag}$ metal decorated $\mathrm{SnO}_{2}$ material to methoxy propanol, three more kinds of VOC gases (also widespread in our life) were configurated for comparison, namely, ethanol, isopropanol and $p$-xylene. Molecular structures of the abovementioned VOC gases are shown in Fig. 11.

For each VOC gas, two typical adsorption configurations on $\mathrm{Ag}$ metal decorated $\mathrm{SnO}_{2}$ (110) surface with oxygen species preadsorbed were simulated, namely, vertical configuration (gas molecule was placed vertically) and parallel configuration (gas molecule was placed parallelly). The two adsorption configurations of methoxy propanol on $\mathrm{SnO}_{2}$ (110) surface are shown in Fig. 12 and the case of the other three gases are similar.

The adsorption energy of four kinds of VOC gases on $\mathrm{Ag}$ metal decorated $\mathrm{SnO}_{2}$ (110) surface with oxygen species pre-

Table 2 Adsorption energy of different gases on oxygen species pre-adsorbed and Ag metal decorated $\mathrm{SnO}_{2}(110) \mathrm{surface}$

\begin{tabular}{|c|c|c|c|c|}
\hline \multirow[b]{2}{*}{ Adsorption energy $(\mathrm{eV})$} & \multicolumn{4}{|l|}{ Target VOC gases } \\
\hline & Methoxy propanol & Ethanol & Isopropanol & $p$-Xylene \\
\hline Vertical & -2.231 & -1.170 & -1.932 & -1.960 \\
\hline Parallel & -3.184 & -1.823 & -0.707 & -1.850 \\
\hline Average value & -2.708 & -1.497 & -1.320 & -1.905 \\
\hline
\end{tabular}


adsorbed were calculated and the results are summarized in Table 2. It could be seen that the absolute value of adsorption energy for methoxy propanol is the largest in the vertical configuration, parallel configuration or the average value, which implies that the adsorption strength of methoxy propanol on $\mathrm{SnO}_{2}$ (110) surface is the strongest.

Transfer charge of different gases on $\mathrm{Ag}$ metal decorated $\mathrm{SnO}_{2}$ (110) surface with oxygen species pre-adsorbed are summarized in Table 3. Compared with the data in Table 1, the negative charge of oxygen species pre-adsorbed in all cases increased after gas adsorption, which states that pre-adsorbed oxygen species obtained more charges. On the other hand, all the four gases lost electrons in every adsorption configuration and the charge transferred from methoxy propanol is the maximum.
The DOS and PDOS spectrogram for the adsorption of methoxy propanol are shown in Fig. 13; it could be seen that three more peaks could be found in the energy range of -13 to $-11 \mathrm{eV}$ (labelled as 1), which was attributed by the adsorbate of methoxy propanol; the DOS value increased in the place labelled as 2. The changes in DOS and PDOS would further account for the increase in the electron energy and hence a better electrical conductivity.

In brief, when methoxy propanol was adsorbed on $\mathrm{Ag}$ metal decorated $\mathrm{SnO}_{2}$ (110) surface with oxygen species pre-adsorbed, the adsorption energy and transfer charge are both the maximum, which implies the strongest adsorption, chemical reaction and therefore the influence on the conductivity of adsorption surface during the adsorption of methoxy propanol gas.

Table 3 Transfer charge of different gases on oxygen species pre-adsorbed and Ag decorated $\mathrm{SnO}_{2}$ (110) surface

\begin{tabular}{|c|c|c|c|c|c|c|c|c|}
\hline (e) & Vertical & Parallel & Vertical & Parallel & Vertical & Parallel & Vertical & Parallel \\
\hline Gases & 0.160 & 0.240 & 0.150 & 0.201 & 0.175 & 0.073 & 0.363 & 0.323 \\
\hline
\end{tabular}
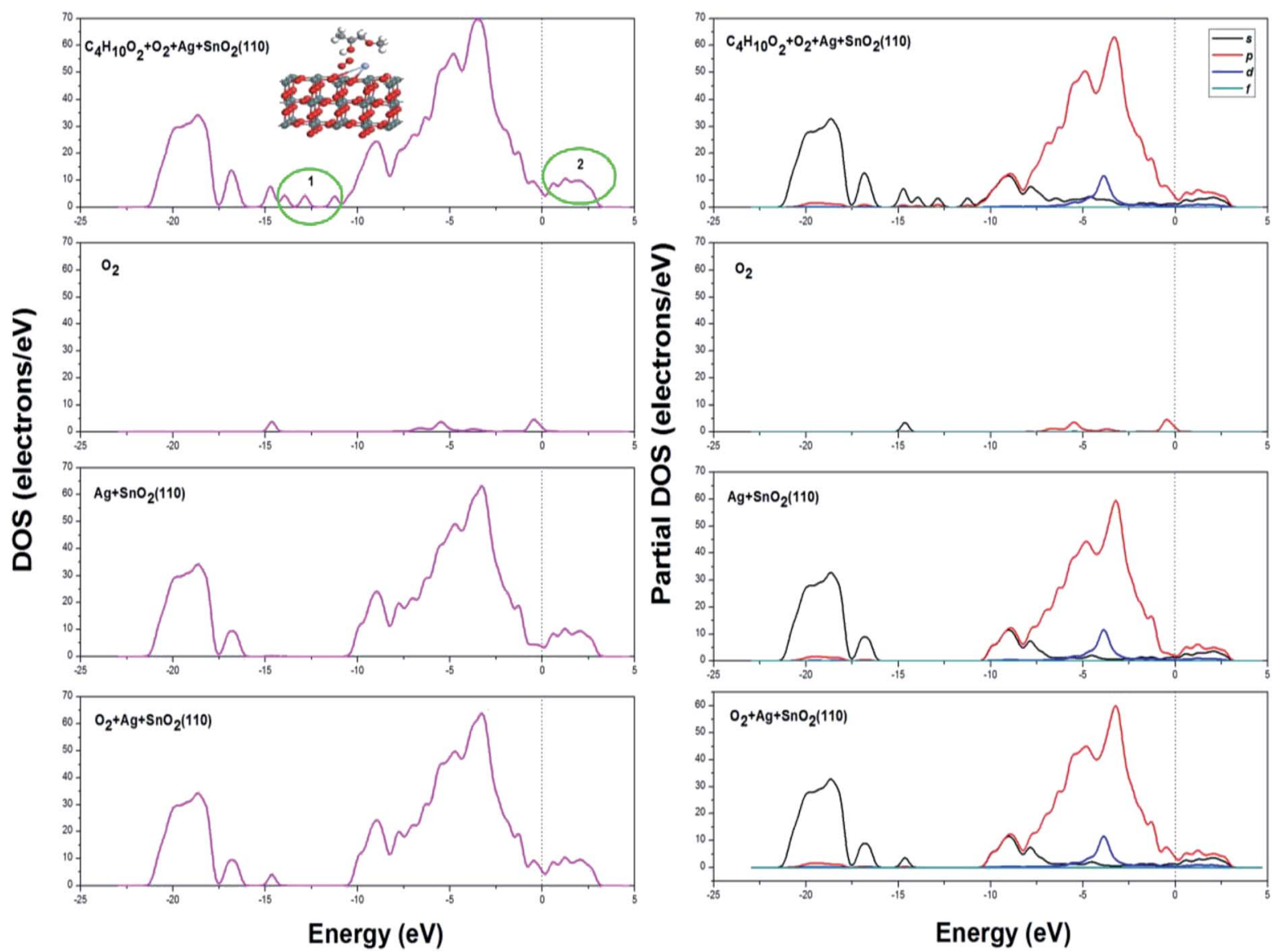

Fig. 13 DOS and PDOS spectrogram after methoxy propanol $\left(\mathrm{C}_{4} \mathrm{H}_{10} \mathrm{O}_{2}\right)$ adsorption on Ag decorated $\mathrm{SnO}$ (110) surface with oxygen species pre-adsorbed. 


\section{Conclusions}

In summary, based on Surface Resistance Controlling Theory of $\mathrm{SnO}_{2}$ gas sensing material, firstly, an electron transfer model on $\mathrm{Ag}$ and $\mathrm{SnO}_{2}$ grain interface was put forward to illustrate the methoxy propanol gas sensing mechanism. Then, a three-layer adsorption model (TLAM) was proposed to investigate the VOC gas sensing mechanism on $\mathrm{Ag}$ decorated $\mathrm{SnO}_{2}$ (110) surface. The calculation results of layer 1 showed that $\mathrm{Ag}$ atom indeed gained negative charges and therefore accumulated more electrons on the surface of $\mathrm{Ag}$ metal decorated $\mathrm{SnO}_{2}$ materials than the case of pure $\mathrm{SnO}_{2}$, which was in good line with the catalytic effect, as mentioned in this paper. In contrast, $\mathrm{Ag}$ atom could get more charge from the $\mathrm{SnO}_{2}$ (110) surface when decorated above $\mathrm{Sn}$ atom than above $\mathrm{O}$ atom. The results of layer 2 showed that the adsorption of molecular oxygen on $\mathrm{Ag}$ metal decorated $\mathrm{SnO}_{2}$ (110) surface was the strongest; molecular oxygen captured the maximum electrons from the material surface when molecular $\mathrm{O}_{2}$ was above the $\mathrm{Ag}$ atom perpendicular to the OA axis. In layer 3 of TLAM, for comparison, three more gases (ethanol, isopropanol and $p$-xylene) were configurated to study the high sensitivity and good selectivity of $\mathrm{Ag}$ metal decorated $\mathrm{SnO}_{2}$ material to methoxy propanol. The results show that the adsorption energy and transfer charge of methoxy propanol are both the maximum among the target gases, which implies the strongest adsorption, chemical reaction and the influence on the conductivity of adsorption surface, and therefore provides more theoretical evidences for high sensitivity and good selectivity of $\mathrm{Ag}$ metal decorated $\mathrm{SnO}_{2}$ materials to methoxy propanol.

\section{Conflicts of interest}

There are no conflicts to declare.

\section{Acknowledgements}

The authors thank Key Research and Development Projects of Yantai (2018XSCC033, 2016ZH053, 2017ZH064); PhD Start-up Fund of Shandong Technology and Business University (BS201810, BS201811); National Natural Science Foundation of China (61804089, 61174007); Natural Science Foundation of Shandong Province (ZR2019MF069); Project of Shandong Province Higher Educational Science and Technology Program (J18KA325, J16LN04) for financial support.

\section{References}

1 K. Andersson, J. V. Bakke and O. Bjørseth, Indoor Air, 1997, 7, 78.

2 A. Dentan, A. Devanthéry and J. E. de Peyer, Int. Arch. Occup. Environ. Health, 2000, 73, 349.

3 A. Muttray, J. Gosepath and J. Brieger, Toxicol. Lett., 2013, 220, 187.

4 Deutsche Forschungsgemeinschaft, The MAK Collection for Occupational Health and Safety, Wiley-VCH, Weinheim, 1998.
5 J. Brieger, A. Muttray and D. Jung, Toxicol. Lett., 2008, 177(2), 138.

6 V. Heinrich, P. S. Cui, H. Pu and E. C. Mattson, Nanoscale, 2012， 4, 5887, http://med.wanfangdata.com.cn/Paper/ Detail/PeriodicalPaper_PM22899322.

$7 \mathrm{M}$. Batzill and U. Diebold, The surface and materials science of tin oxide, Prog. Surf. Sci., 2005, 79, 47.

8 X. C. Ma, H. Y. Song and C. S. Guan, Sens. Actuators, B, 2013, 177, 196.

9 F. Gyger, M. Hübner and C. Feldmann, Chem. Mater., 2010, 22, 4821.

10 D. Ju, H. Xu and X. Qi, Sens. Actuators, B, 2015, 215, 39.

11 M. Li, W. Yan and H. Zhu, RSC Adv., 2015, 5, 51953.

12 L. Liu, C. Guo and S. Li, Sens. Actuators, B, 2010, 150, 806.

13 J. Aguilar-Leyva, A. Maldonado and M. de la L. Olvera, Mater. Charact., 2007, 58, 740.

14 Y. V. Kaneti, J. Yue and J. Moriceau, Sens. Actuators, B, 2015, 219, 83.

15 T. T. Wang, S. Y. Ma and L. Cheng, Sens. Actuators, B, 2015, 216, 212.

16 L. Mädler, T. Sahm and A. Gurlo, J. Nanopart. Res., 2006, 8, 783.

17 J. Oviedo and M. J. Gillan, Surf. Sci., 2001, 490, 221.

18 F. R. Sensato, R. Custódio and M. Calatayud, Surf. Sci., 2002, 511, 408.

19 Y. B. Xue and Z. A. Tang, Sens. Actuators, B, 2007, 20, 2364.

20 A. V. Bandura, J. D. Kubicki and J. O. Sofo, J. Phys. Chem. B, 2008, 112, 11616.

21 J. B. L. Martins, E. Longo and J. Andres, J. Mol. Struct.: THEOCHEM, 1995, 335, 167.

22 M. MelleFranco and G. Pacchioni, Surf. Sci., 2000, 461, 54.

23 F. Ciriaco, L. Cassidei and G. Petrella, Chem. Phys., 2004, 303, 55.

24 Y. B. Xue and Z. A. Tang, Sens. Actuators, B, 2009, 138, 108.

25 J. D. Prades, A. Cirera and J. R. Morante, Sens. Actuators, B, 2007, 126, 62.

26 G. Xu, L. Zhang and C. He, Sens. Actuators, B, 2015, 221, 717.

27 M. Calatayud, J. Andrés and A. Beltrán, Surf. Sci., 1999, 430, 213.

28 J. Yue, X. C. Jiang and A. B. Yu, J. Phys. Chem. C, 2013, 117, 9962.

29 W. Zhao, F. H. Tian and X. Wang, J. Colloid Interface Sci., 2014, 430, 18.

30 F. H. Tian, C. Gong and Y. Peng, Sens. Actuators, B, 2017, 244, 655.

31 M. Li, H. Zhu and B. Wang, Sens. Actuators, B, 2016, 232, 545. 32 D. Williams, Sens. Actuators, B, 1999, 57, 1.

33 N. Bârsan, M. Schweizer-Berberich and W. Göpel, Fresenius. J. Anal. Chem., 1999, 365, 287.

34 W. Göpel and K. D. Schierbaum, Sens. Actuators, B, 1995, 26, 1.

35 S. Cui, H. Pu and E. C. Mattson, Nanoscale, 2012, 4, 5887, http://med.wanfangdata.com.cn/Paper/Detail/

PeriodicalPaper_PM22899322.

36 I. S. Hwang, J. K. Choi and H. S. Woo, ACS Appl. Mater. Interfaces, 2011, 3, 3140. 
37 H. Wang, Q. Liang and W. Wang, Cryst. Growth Des., 2011, 11, 2942.

38 S. Liu, M. Xie and Y. Li, Sens. Actuators, B, 2010, 151, 229.

39 B. Delley, J. Chem. Phys., 2000, 113, 7756.

40 B. Delley, J. Chem. Phys., 1990, 92, 508.

41 J. P. Perdew, K. Burke and M. Ernzerhof, Phys. Rev. Lett., 1996, 77, 3865.

42 R. S. Mulliken, Chem. Phys., 1955, 23(12), 1833.

43 B. Cheng, J. M. Russell and W. S. Shi, J. Am. Chem. Soc., 2004, 126(19), 5972.
44 D. F. Zhang, L. D. Sun and J. L. Yin, Adv. Mater., 2003, 15(12), 1022.

45 J. M. Themlin, M. Chtaïb and L. Henrard, Phys. Rev. B: Condens. Matter Mater. Phys., 1992, 46(4), 2460.

46 J. Goniakowski and M. J. Gillan, Surf. Sci., 1996, 350, 145.

47 M. Viitala, O. Cramariuc and B. Delley, Surf. Sci., 2011, 605, 1563.

48 W. Zeng, T. Liu and D. Liu, Phys. B, 2010, 405, 4235. 